\title{
Sensitivity of narrow- and wideband LNA performance to individual transistor model parameters
}

\author{
Marnus Weststrate ${ }^{\mathrm{a} *}$, Anindya Mukherjee $^{\mathrm{b}}$, Saurabh Sinha $^{\mathrm{a}}$ and Michael Schröter ${ }^{\mathrm{b}}$ \\ ${ }^{a}$ Department of Electrical, Electronic and Computer Engineering, University of Pretoria, \\ Pretoria, South Africa; ${ }^{b}$ Chair for Electron Devices and Integrated Circuits, Dresden University \\ of Technology, Dresden, Germany
}

\begin{abstract}
Although it is desirable for a transistor model to be as accurate as possible the extraction of model parameters from fabricated transistors is a time consuming and often costly process. An investigation of the sensitivity of LNA performance characteristics to individual parameters of the physics-based standard HBT model HICUM/L2 was therefore done to gain preliminary insight into the most important parameters for transistors used in actual circuits. This can potentially allow less strenuous accuracy requirements on some parameters which would ease the extraction process. Both a narrow- and a wideband LNA configuration were investigated. It was found that the series resistance parameters have a large impact on LNA gain, $S_{11}$ and NF performance in both cases. Since the narrow band LNA relies heavily on the transistor characteristics to provide proper matching it was also very sensitive to changes in the parameters used in modelling the high frequency current gain and depletion capacitances of the transistor.
\end{abstract}

Keywords: sensitivity; low noise amplifier; inductive emitter degeneration; LC-ladder and capacitive feedback; parameter variations; HICUM; solid-state electronics devices

\section{Introduction}

Transistor models are evaluated on how accurately they reflect the measured characteristics of

fabricated transistors. It is desirable for these models to be as accurate as possible; however, extraction of model parameters from fabricated transistors is a time consuming and often costly process. The more physical effects a transistor model covers, the greater the number of model parameters to extract usually becomes. The latest level 2 high current model (HICUM/L2 [1]) for example has 114 model parameters.

This complexity creates a need for knowing how inaccuracies in model parameters affect the most important characteristics of actual circuits. This could potentially save much time and money by allowing less strenuous accuracy specifications for parameters involved in modelling characteristics which do not have a large effect on actual circuit performance. Apart from this, such a study provides vital feedback to the model developers regarding the importance of a group of model parameters with a significant impact on a particular kind of circuit.

An initial study was undertaken to investigate the sensitivity of the most important low noise amplifier (LNA) performance characteristics, namely the gain $\left(S_{21}\right)$, input reflection 
coefficient $\left(S_{11}\right)$ and noise figure $(\mathrm{NF})$, when simulated using HICUM. Both a wideband and narrowband configuration were investigated using the HICUM level 2 v2.24 transistor model shown in Figure 1.

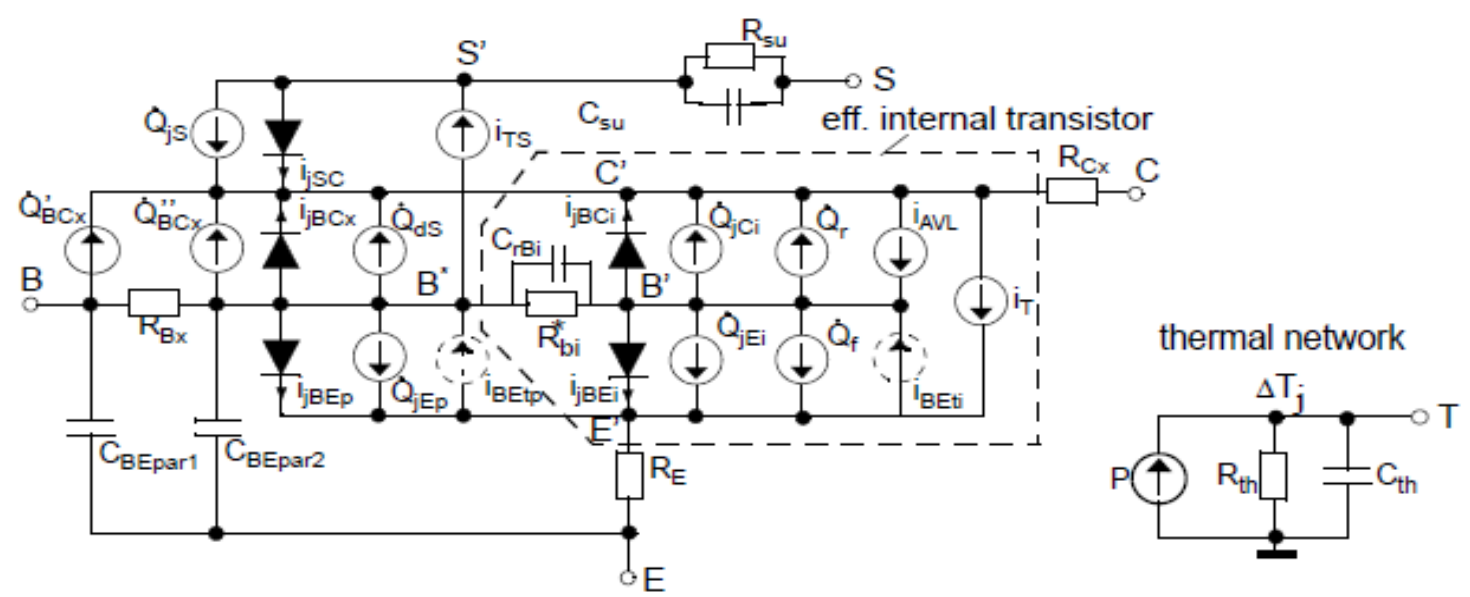

vertical NQS effects
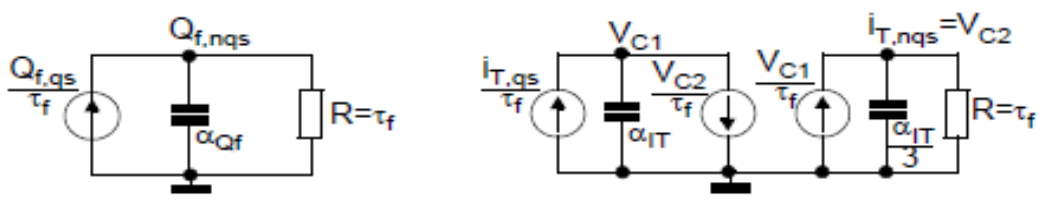

Figure 1. The HICUM level 2 v2.24 transistor model used to simulate all presented results.

Varying each model parameter in turn and comparing the resulting performance to the reference case over the frequency band of interest the sensitivity of these characteristics to the individual model parameters were found. These results are presented in the form of bar graphs showing the sensitivity of these characteristics to variations in the model parameters in a comparative format.

A SiGe process with an $f_{T}$ of $50 \mathrm{GHz}$ has been considered in this work. The required HICUM parameters were generated with the integrated modelling tool TRADICA [2], which is an expert system allowing for the quick generation of consistent compact model parameters based on process information and device configuration. 


\section{LNA configurations and characteristics}

To gain a more extensive understanding of the sensitivity of LNAs to model parameter variations both a narrowband and a wideband configuration was used. These are discussed briefly in the following subsections.

\subsection{Inductive emitter degeneration}

The inductive emitter degeneration technique is well known and has become possibly the most frequently implemented LNA configuration since its introduction [3]. The advantage of using this technique is that it is theoretically possible to obtain a simultaneous optimal noise and conjugate input match through proper selection of the emitter length and designing the equivalent RLC circuit at the input to resonate at the operating frequency by choosing the values of the added emitter and base series inductances [3].

The schematic and its equivalent circuit are shown in Figure 2. The input impedance of the LNA is given by [4]:

$$
Z_{I N}=\frac{g_{m} L_{E}}{C_{\pi}}+j \omega L_{E}+\frac{1}{j \omega C_{\pi}}=\underbrace{\omega_{T} L_{E}}_{R_{e f f}}+j \omega L_{E}+\frac{1}{j \omega C_{\pi}} .
$$

The forward gain can be derived from the voltage gain which is the combination of the gain through the input matching network and the gain of the transistor and is given by

$$
\begin{aligned}
S_{21} & =2 \cdot A_{v, I M N} \cdot G_{M} Z_{L} \\
& =2 \cdot \frac{Z_{E Q}}{\left(Z_{E Q}+Z_{L B}+R_{S}\right)} \cdot \frac{g_{m} Z_{L}}{\left(1+g_{m} Z_{L E}\right)},
\end{aligned}
$$

where $Z_{E Q}$ is the equivalent RLC circuit seen looking into the base of the transistor and $Z_{L B}$ and $Z_{L E}$ are the impedances of the inductors including a parasitic series resistance. Following a derivation similar to the one in [6] the noise factor can be represented by

$$
F=1+\frac{\overline{v_{L B}^{2}}+\overline{v_{L E}^{2}}+\overline{v_{c e}^{2}}+\left|Z_{L B}+R_{S}\right|^{2} \cdot \overline{i_{c e}^{2}}}{\overline{v_{S}^{2}}}
$$


where $v_{L B}$ and $v_{L E}$ are the noise generators of the parasitic resistance in the inductors and $v_{c e}$ and $i_{c e}$ are the well known common-emitter transistor noise sources. In (1b) and (1c) $C_{\mu}$ has been neglected consistent with the derivation of the input impedance in [4].

A LNA was designed to operate at $5 \mathrm{GHz}$ and achieved a simulated gain of $16 \mathrm{~dB}, S_{11}$ of less than $-12 \mathrm{~dB}$ and maximum $\mathrm{NF}$ of $3 \mathrm{~dB}$ using this configuration.

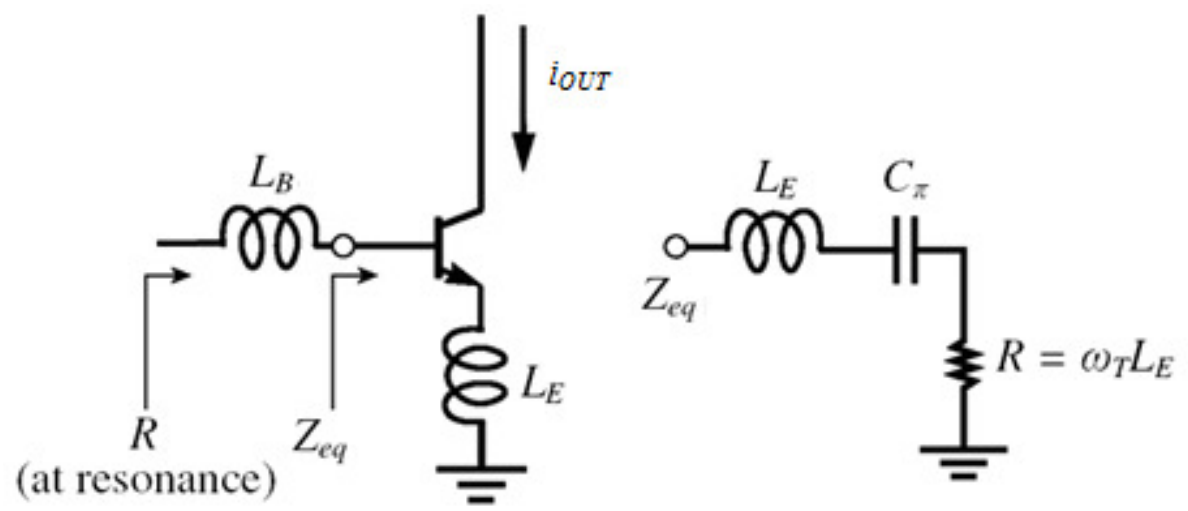

Figure 2. High frequency equivalent circuit at the base of an inductively degenerated commonemitter amplifier [5].

\subsection{LC-ladder and capacitive shunt-shunt feedback}

The LC-ladder and capacitive shunt-shunt feedback configuration shown in Figure 3 is a relatively new LNA configuration introduced recently [6] in an attempt to overcome certain shortcomings identified in conventional wideband LNA implementations [7]. The configuration relies on a fourth order band-pass filter at the input to realize a desired equivalent input impedance over a theoretically arbitrary bandwidth. The series capacitance and resistance in the latter part of this ladder filter results from the Miller-impedance of the feedback capacitance and load resistance in the first stage.

The simplified expressions for the characteristics under investigation, where $Z_{L 1}$ and $Z_{L 2}$ are the impedances of the inductors including a series parasitic resistance, are [6]:

$$
\begin{gathered}
\left.Z_{I N} \approx j \omega L_{1}\left\|\frac{1}{j \omega C_{1}}\right\|\left(j \omega L_{2}+\frac{1}{j \omega\left[C_{\pi 1}+\left(1+g_{m 1} R_{L 1}\right)\left(C_{\mu 1}+C_{F}\right)\right.}\right]+\frac{1}{g_{m 1}}\left[1+\frac{C_{L 1}}{C_{\mu 1}+C_{F}}\right]\right), \\
S_{21}=\frac{2 Z_{S} R_{L 1}\left(R_{S}+\frac{1}{j \omega C_{2}}\right)\left(g_{m 1}-j \omega C_{\mu 1}-j \omega C_{F}\right)\left(j \omega L_{3} g_{m 2}\right)}{R_{S}\left(Z_{S}+j \omega L_{2}+\frac{1}{j \omega C_{2}}+R_{S}\right)\left(1+j \omega R_{L 1}\left(C_{\mu 1}+C_{F 1}+C_{L 1}\right)\right)},
\end{gathered}
$$




$$
\begin{gathered}
F=1+\left[\left(\frac{1}{\left|Z_{S}\right|^{2}}+\left|1+\frac{Z_{L 2}}{Z_{S}}\right|^{2} \cdot\left|\omega C_{F}\right|^{2}\right) \overline{v_{c e}^{2}}+\left|1+\frac{Z_{L 2}}{Z_{S}}\right|^{2} \overline{i_{c e}^{2}}+\frac{\overline{v_{L 1}^{2}}}{\left|Z_{L 1}\right|^{2}}+\frac{\overline{v_{L 2}^{2}}}{\left|Z_{S}\right|^{2}}\right] \cdot \frac{R_{S}^{2}}{\overline{v_{R S}^{2}}}, \\
\text { where } Z_{S}=\left(\frac{1}{R_{S}}+\frac{1}{Z_{L 1}}+j \omega C_{1}\right)^{-1} .
\end{gathered}
$$

A LNA operating over the arbitrary band from $0.8 \mathrm{GHz}$ to $6 \mathrm{GHz}$ was designed with a simulated gain of $18 \mathrm{~dB}$, an $S_{11}$ less than $-8 \mathrm{~dB}$ and maximum NF of $4.5 \mathrm{~dB}$ using the design equations presented in [6] and the noise optimization method from [8].

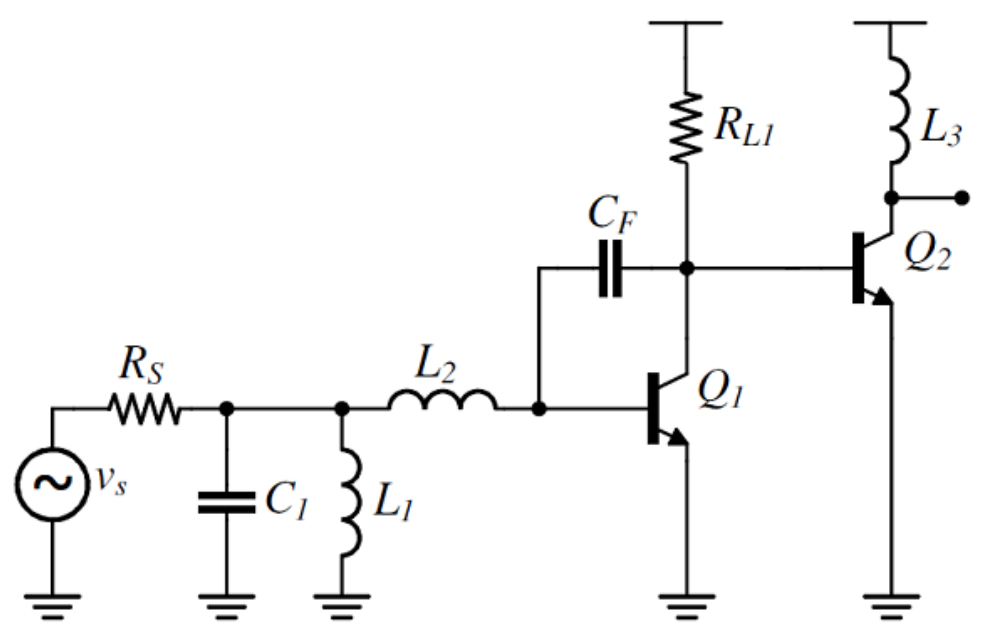

Figure 3. Schematic of the LC-ladder and capacitive shunt-shunt feedback LNA configuration. The inductive load of the second stage is used to flatten the gain [6].

\section{Methodology}

The simulations were done using the Cadence Virtuoso analogue environment. The latest version 2.24 of HICUM/L2 was used with device emitter dimensions of $0.2 \mu \mathrm{m} \times 12 \mu \mathrm{m}$. An OCEAN script was used to automate the variation of the individual model parameters. Each model parameter was varied by $\pm 10 \%$ and $\pm 20 \%$ and the frequency sweep of the $S_{21}, S_{11}$ and NF characteristics were recorded.

Subsequently post processing was done in Matlab. The deviation $(\Delta)$ of the response $($ data $)$, due to one of the four variations $(v)$ in a parameter $(p)$, from the reference case (ref) was calculated over the frequency band of interest using

$$
\Delta_{p, v}(f)=\frac{\mid r e f(f)-\text { data }_{p, v}(f) \mid}{\text { normalization_factor }_{\text {farma }}}
$$


where the normalization factor is one for the case of $\pm 10 \%$ deviation and two for $\pm 20 \%$ deviation. Five frequency points at equal intervals over the frequency band of interest were selected and the sensitivity $\left(\sigma^{\prime}\right)$ at these frequencies calculated as the average deviation from the reference as a percentage using

$$
\sigma_{p}^{\prime}\left(f_{n}\right)=\frac{\sum_{v=1}^{4} \Delta_{p, v}\left(f_{n}\right)}{4 \cdot \operatorname{ref}\left(f_{n}\right)} \times 100 .
$$

The final deviations given in the results are the averages of the deviations calculated at these five selected frequencies given by

$$
\sigma_{p}=\frac{\sum_{n=1}^{5} \sigma_{p}^{\prime}\left(f_{n}\right)}{5} .
$$

\section{Results}

The simulated gain $\left(S_{21}\right)$, input reflection coefficient $\left(S_{11}\right)$ and NF plots of the two LNAs were investigated. The following subsections present a comparison of the sensitivity between the parameters for which the sensitivity was the highest.

\subsection{Inductive emitter degeneration LNA}

Since the input matching of the emitter degenerated configuration depends largely on the transistor characteristics, most notably the emitter length, current gain and base emitter capacitance, the performance of this LNA, and especially the $S_{11}$, is very sensitive to transistor model parameter variations. The variations in the performance of this configuration are shown in Figure 4. 


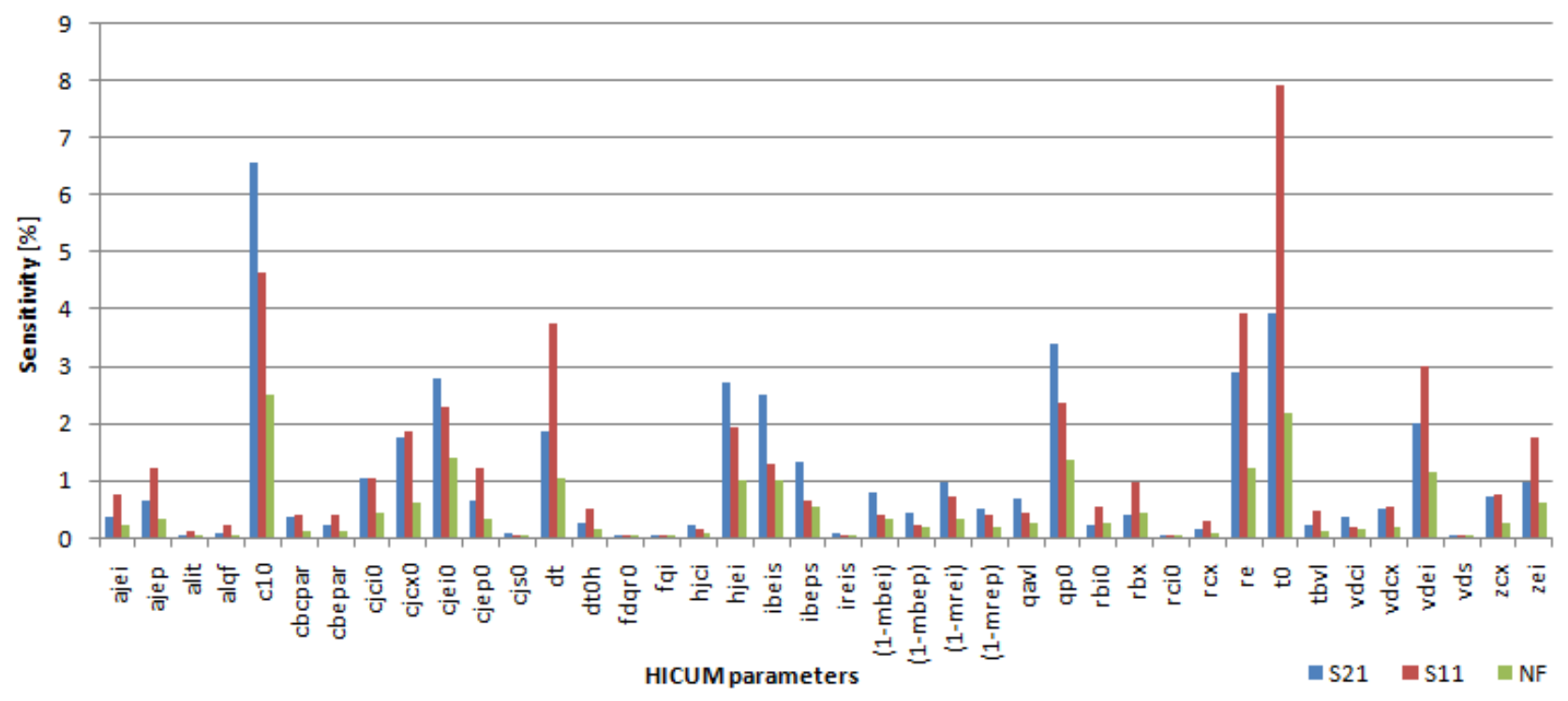

Figure 4. Comparison of the sensitivity of the inductively emitter degenerated LNA performance to the most sensitive HICUM model parameters.

The input impedance of the LNA is given by [3]

$$
\begin{aligned}
Z_{I N} & =j \omega L_{B}+j \omega L_{E}\left(1+\beta_{R F}\right)+\frac{1}{j \omega C_{\pi}} \\
& \approx j \omega L_{B}+j \omega L_{E}\left(1-j \frac{\omega_{T}}{\omega}\right)+\frac{1}{j \omega C_{\pi}}, \\
& =j \omega\left(L_{B}+L_{E}\right)+\frac{1}{j \omega C_{\pi}}+\omega_{T} L_{E}
\end{aligned}
$$

which represents the equivalent series RLC circuit shown in Figure 2 including the inductor $L_{B}$. Equation (6a) indicates this dependence of $S_{11}$ on the total base-emitter capacitance $\left(C_{\pi}\right)$ and the transit frequency $f_{T}=\omega_{T} /(2 \pi)$ via the high frequency $(\mathrm{HF})$ transistor current gain $\left(\beta_{R F}\right)$. Changes in these values will cause the resonant frequency where a conjugate match is obtained to shift, and a change in the HF current gain also moves the equivalent resistance seen at this frequency away from $50 \Omega$.

Figure 4 shows that the vdei parameter, describing the internal base emitter built-in potential, causes a large change in the input matching compared to the corresponding base collector built-in potential parameter, $v d c i$, by translating directly into a change in the capacitance of the equivalent input RLC circuit. The series resistance parameters, and especially the $\beta$-multiplied emitter resistance $\left(r_{e}\right)$, are also important as they are in series with this equivalent RLC circuit at the 
base of the transistor and add to the equivalent resistance seen at the resonant frequency which is in fact given by

$$
Z_{I N} \approx j \omega L_{B}+j \omega L_{E}\left(1-j \frac{\omega_{T}}{\omega}\right)+\frac{1}{j \omega C_{\pi}}+\left(r_{b}+\left(1+\beta_{R F}\right) r_{e}\right)
$$

The deviations caused by vdei and re are shown in Figure 5.
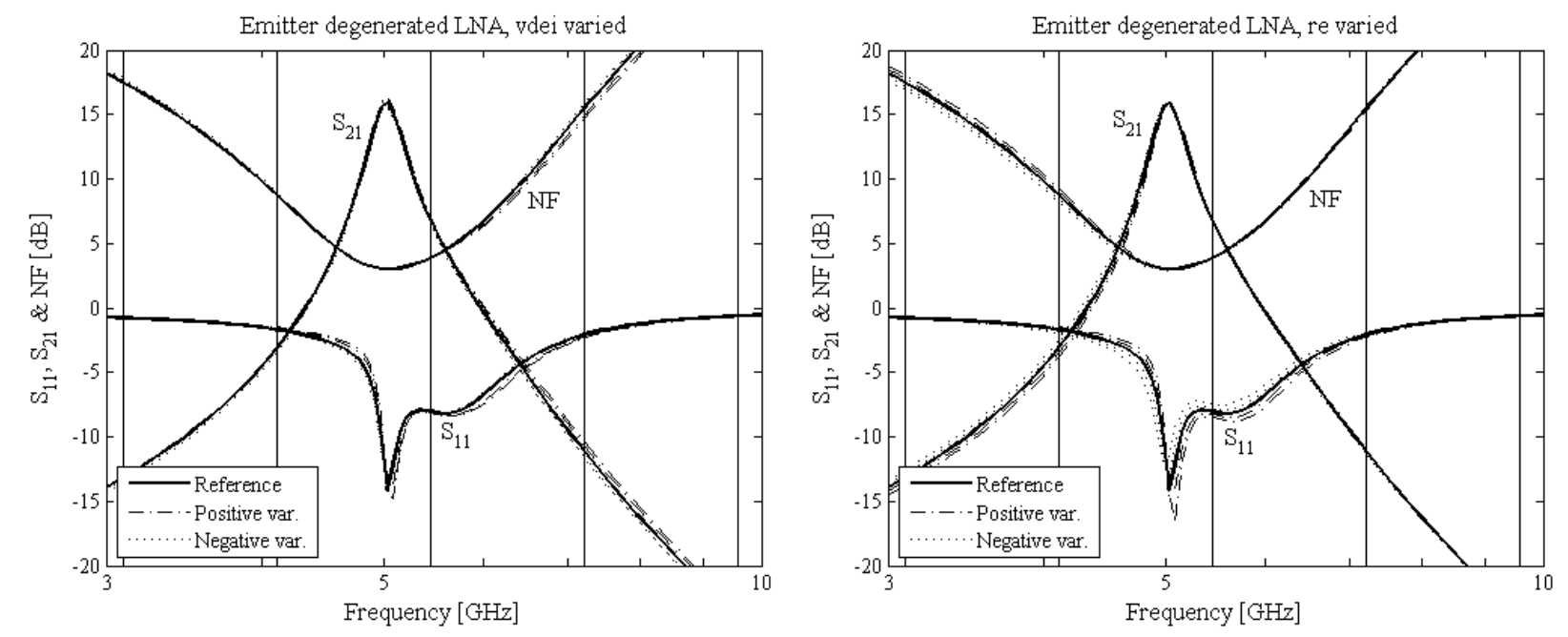

Figure 5. Simulated gain, $S_{11}$ and NF results of the inductively emitter degenerated LNA $\left(f_{0}=5 \mathrm{GHz}\right)$ showing the variations with $v d e i$ and $r e$.

Variations in the ideality factors mbei, mbep, mrei and mrep result in a very large direct change in corresponding diode currents. Since it is unlikely that these ideality factors will be extracted with inaccuracies as large as $\pm 10 \%$, the effect of variations in the final $(m-1)$ value are shown instead. This gives a better indication of the effect of reasonable extraction errors for these parameters, which is seen to be relatively small.

Since the parameter $m C f$ is physically supposed to be 1 it was not varied. An exception to this rule are GaAs HBTs when the GICCR based transfer current relation alone is no longer accurate. For the investigated $50 \mathrm{GHz}$ process the value should however be 1 , and deviations in $g_{m}$ are captured properly by changes in $c 10$ and $q p 0$.

Modelling of the minority charge is an important aspect in bipolar transistors. In HICUM the operating point dependent minority charge stored in a forward biased (vertical) transistor can be determined from the device transit time $\left(\tau_{f}\right)$ by the simple integration 


$$
Q_{f}=\int_{0}^{i_{T f}} \tau_{f} d i,
$$

which shows the direct link between transit time and the minority charge. Figure 4 and Figure 6 demonstrates the same showing the very large effect of $t 0$, the low current forward transit time, on the input matching.

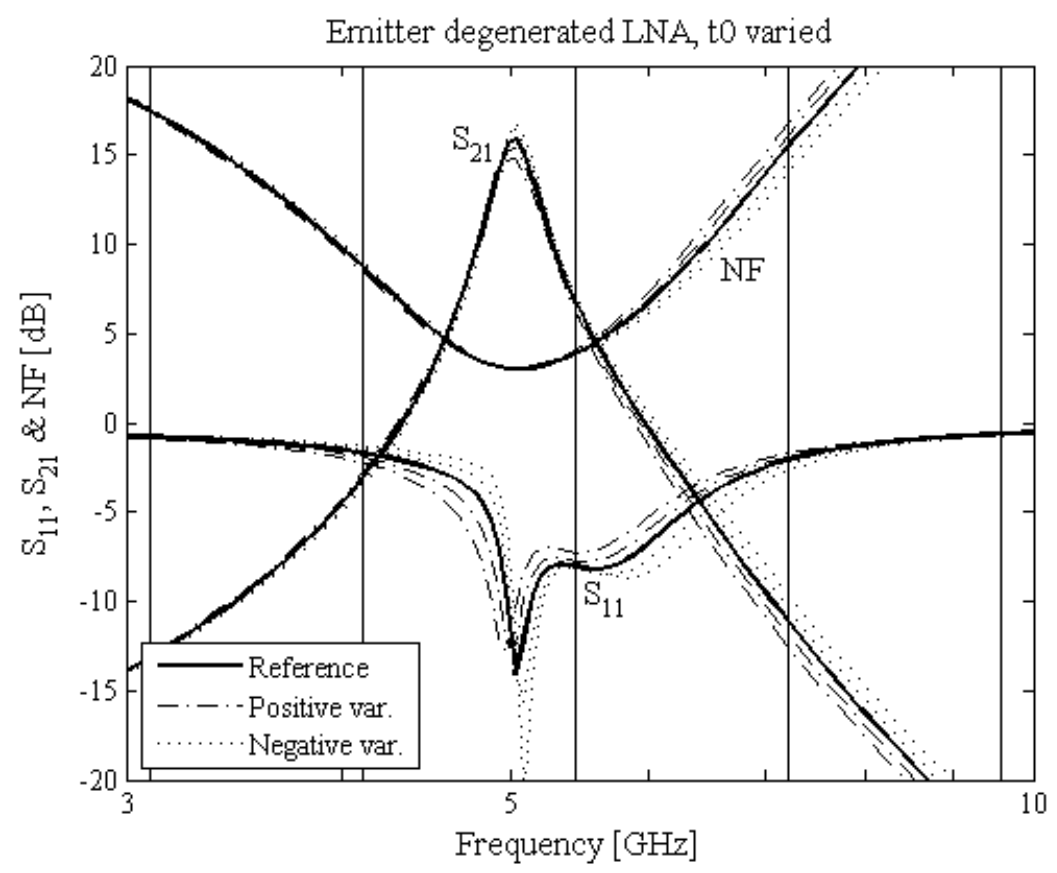

Figure 6. Simulated gain, $S_{11}$ and NF results of the inductively emitter degenerated LNA $\left(f_{0}=5 \mathrm{GHz}\right)$ showing the variations with $t 0$.

Other important HICUM parameters are $c 10$, which is related to the saturation current $\left(I_{s}\right)$ by the relation $c 10=I_{s} * q p 0$, and $q p 0$, which is the zero-bias hole charge of the transistor. These parameters change the transconductance and base-emitter diffusion capacitance. Their effect on transistor performance is especially relevant to the $S_{11}$ and $S_{21}$ as shown in Figure 4 and Figure 7. 

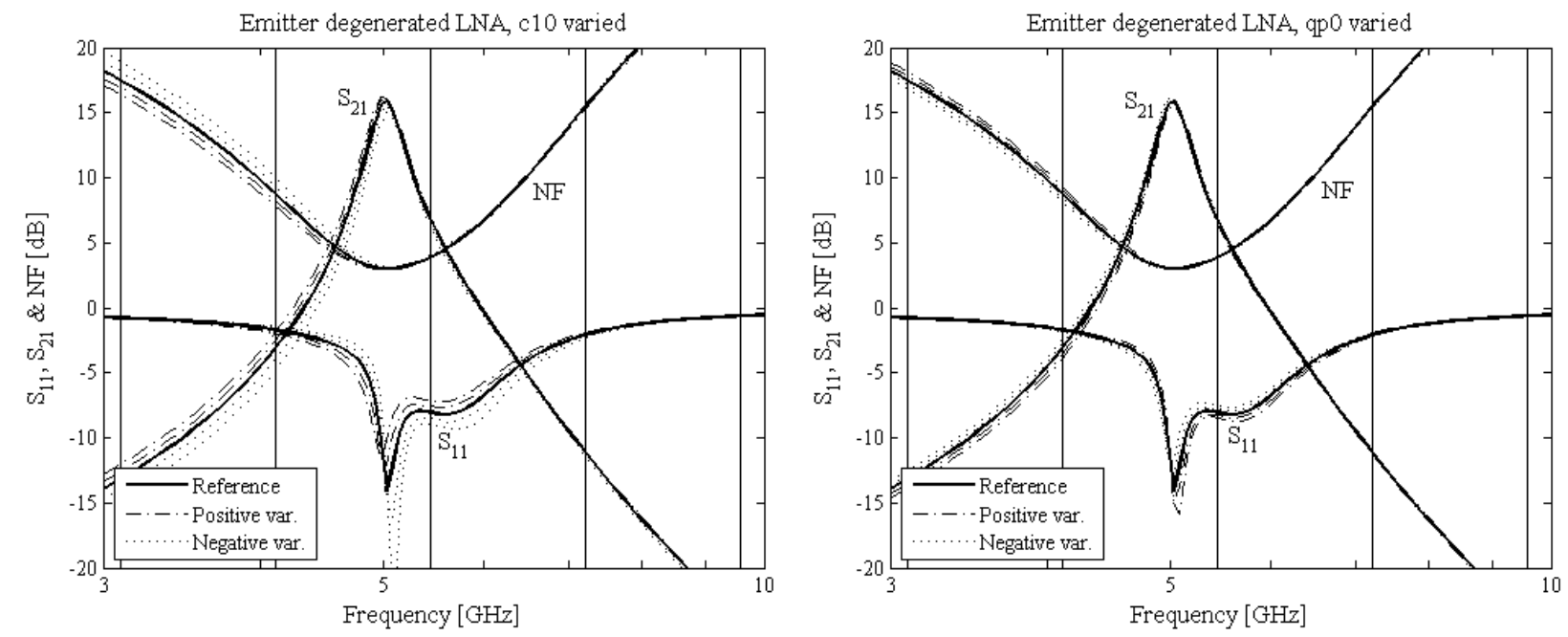

Figure 7. Simulated gain, $S_{11}$ and NF results of the inductively emitter degenerated LNA $\left(f_{0}=5 \mathrm{GHz}\right)$ showing the variations with $c 10$ and $q p 0$.

The base-emitter and base-collector related capacitance components cjciO, cjcxO, cjeiO and cjepO also cause significant deviation; the most discernable of these deviations are caused by cjeiO and $\operatorname{cjcx} 0$ which are shown in Figure 8 . The effect of the base-emitter capacitance parameters is again explained by their relevance to the resonant frequency of the equivalent RLC circuit. The cause of the change with variation in the base-collector capacitance however is not apparent from the design equations since $C_{\mu}$ is neglected in their derivation. While this simplifies the design it has been pointed out that the presence of a Miller-capacitance at the input does have a significant influence on the matching [9] which is corroborated by these results.
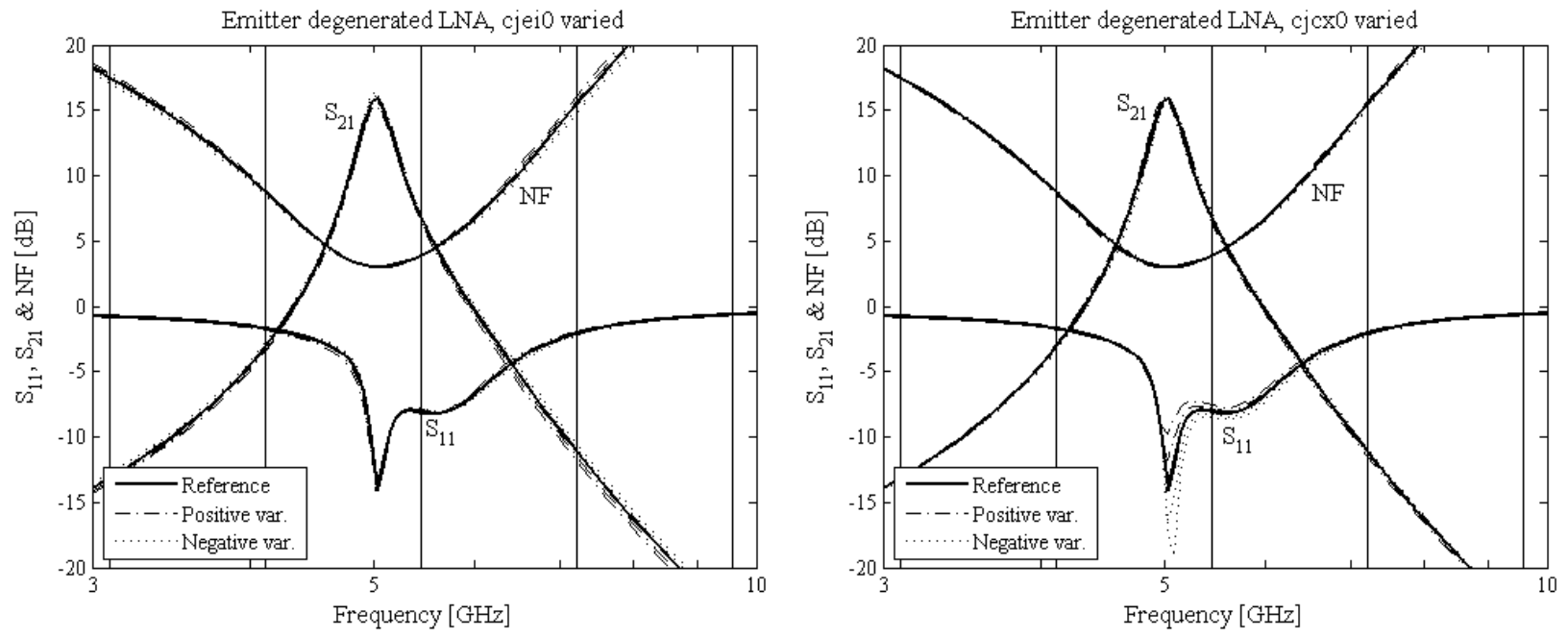

Figure 8. Simulated gain, $S_{11}$ and NF results of the inductively emitter degenerated LNA $\left(f_{0}=5 \mathrm{GHz}\right)$ showing the variations with $c j e i 0$ and $c j c x 0$. 
The gain is mostly affected by the same parameters as the $S_{11}$, albeit to a much smaller extent. The NF shows again smaller deviations, and is affected most by the series resistance parameters $r b i 0, r b x$ and $r e$, which causes thermal noise in the transistor.

The variation in circuit performance with temperature is seen from the variation of the model parameter $d t$ which describes the temperature change in a particular transistor with respect to the chip temperature.

\subsection{LC-ladder and capacitive shunt-shunt feedback LNA}

The LC-ladder and capacitive shunt-shunt feedback configuration is much less dependent on the transistor characteristics since the input matching is largely determined by the LC-ladder and the feedback capacitor value. Comparing Figure 4 and Figure 9 shows that the maximum sensitivity of this configuration is approximately four times smaller than that of the emitter degenerated LNA, and apart from the base and emitter resistances few parameters show a sensitivity of more than $0.3 \%$. Figure 10 shows the performance of the reference case and the deviations due to the re model parameter which is the only parameter that results in a discernable deviation.

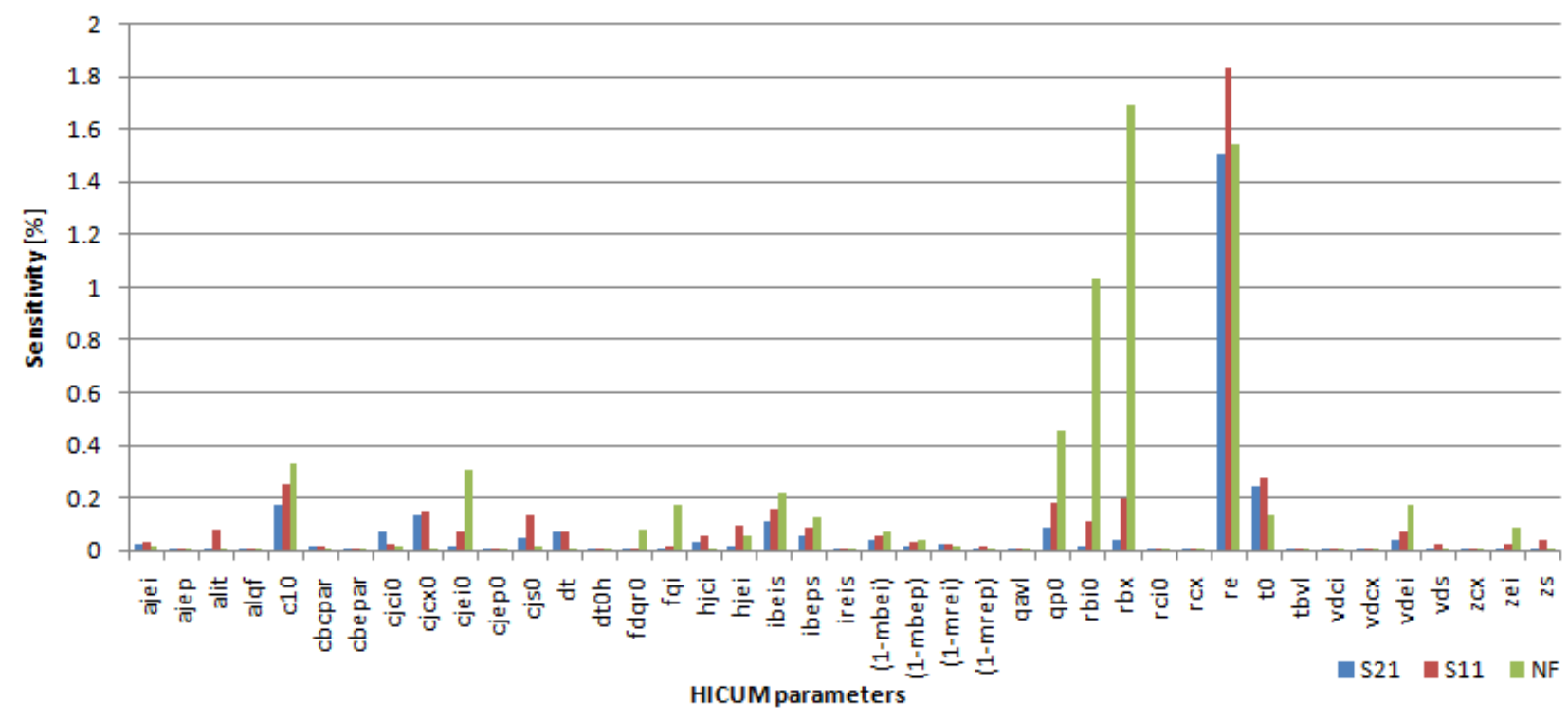

Figure 9. Comparison of the sensitivity of the LC-ladder and capacitive shunt-shunt feedback LNA performance to the most sensitive HICUM model parameters. 


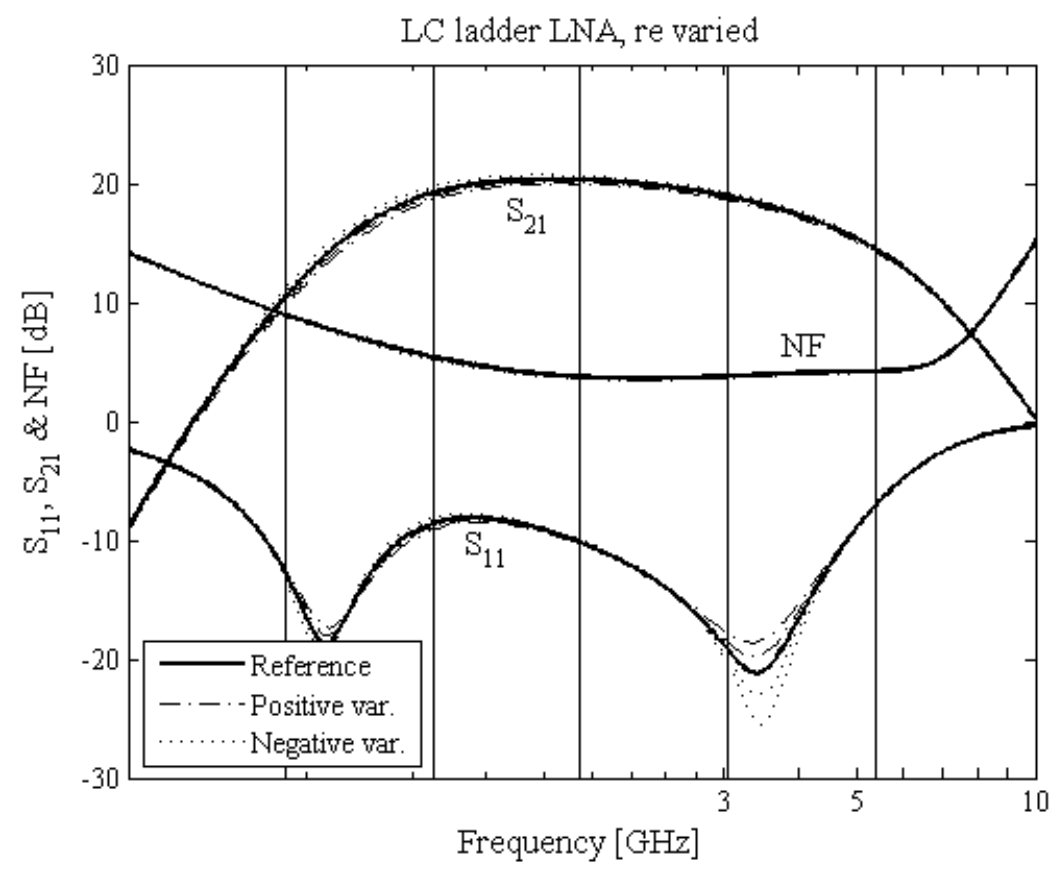

Figure 10. Simulated gain, $S_{11}$ and NF results of the LC-ladder and capacitive shunt-shunt feedback LNA operating over the $0.8 \mathrm{GHz}$ to $6 \mathrm{GHz}$ band with variations caused by $r e$.

The frequency range over which a conjugate match is achieved is determined by the passive components making up the LC-ladder filter according to [6]

$$
\begin{gathered}
L_{1} \approx \frac{R_{S}}{2 \pi f_{L}} \quad \text { and } \quad C_{2} \approx \frac{1}{2 \pi f_{L} R_{S}}, \\
L_{2} \approx \frac{R_{S}}{2 \pi f_{H}} \quad \text { and } \quad C_{1} \approx \frac{1}{2 \pi f_{H} R_{S}},
\end{gathered}
$$

where $f_{H}$ and $f_{L}$ denote the high and low corner frequencies of the matched band. Since all these passives except $C_{2}$ are actual passive components (refer to Figure 3) the input matching is largely independent of variations in the transistor parameters. The series capacitance $\left(C_{2}\right)$ and the equivalent input resistance seen over the matched band are derived from the equivalent Miller-impedance given by [6]

$$
Z_{M}=\frac{1}{j \omega \underbrace{C_{B C}\left(1+g_{m} R_{L}\right)}_{C_{M}}}+\underbrace{\frac{R_{L}}{1+g_{m} R_{L}}\left(1+\frac{C_{L}}{C_{B C}}\right)}_{R_{M}} .
$$

In (9) $C_{B C}$ is the parallel combination of the added feedback capacitance $\left(C_{F}\right)$ and the intrinsic base-collector capacitance $\left(C_{\mu}\right)$ and $g_{m}$ is the transconductance of the first transistor. $C_{L}$ is the total 
node capacitance at the output of the first stage excluding $C_{B C}$ but including other parasitic capacitances and any capacitance added to tune the input matching. Complete design equations can be found in [6] and [8].

Figure 9 shows that the parameters relating to the depletion capacitances such as $v d e i, c j c x O$ and cjeiO have a very small influence on the performance, especially compared to the emitter degenerated case. Although these parasitics are absorbed in the design of the matching network, $C_{F}$ is typically much larger than $C_{\mu}$ and $C_{M}$ dominates in the parallel combination with the base-emitter capacitance $\left(C_{\pi}\right)$ to realize $C_{2}$; this reduces the sensitivity to changes in the intrinsic capacitances.

Since the matching does not rely on the beta-multiplication of an added emitter impedance this configuration is also less sensitive to changes in the base transit time, modelled by $t 0$, and other HF current gain related parameters. The overall gain however does remain important in generating the required Miller-impedance in (9) and as such these parameters have some impact on the performance.

The parameters showing the highest sensitivity are those describing the series resistances. This is especially true of the NF since it is affected most by the equivalent common-emitter voltage noise [8] which is generated by the series resistances at the base (modelled by $r b x$ and $r b i 0$ ) and emitter (modelled by re) according to [10]

$$
\overline{v_{C E}^{2}}=4 k T\left(r_{b}+r_{e}+\frac{1}{2 g_{m}}\right) \text {. }
$$

Since the emitter resistance is beta-multiplied and in series with the series part of the equivalent input circuit, it has the largest effect on input matching of all parameters in Figure 9; together with the base resistance it changes the equivalent input resistance to

$$
R_{I N} \approx \frac{R_{L}}{\left(1+g_{m} R_{L}\right)}\left(1+\frac{C_{L}}{C_{B C}}\right)\left(\frac{C_{M}}{C_{\pi}+C_{M}}\right)^{2}+(1+\beta) r_{e}+r_{b} .
$$

The emitter resistance also affects the gain due to the emitter degeneration it provides. 


\section{Conclusion}

The sensitivities of an inductively degenerated LNA and a LC-ladder and capacitive feedback LNA to the individual HICUM parameters have been investigated. Varying the parameters used in modelling the depletion capacitances and high-frequency current gain had a large impact on the emitter degenerated LNA since its design relies on leveraging the transistor characteristics. In the LC-ladder and capacitive feedback case these were, however, less important as its design relies more on the passive components in the ladder filter and feedback network.

The series resistance parameters had a large impact on both LNA configurations. In the case of the emitter degenerated LNA the effect on the input matching was most prominent as these resistances occur in series with the input impedance. In the LC-ladder and capacitive feedback case the NF showed the largest sensitivity since the NF is dominated by the transistor voltage noise.

This work indicates that the LNA topology selected for a particular application is very important with regards to sensitivity. One might, for example, expect a narrow-band LNA such as the one in [4], which also relies on a Miller-impedance to realize matching, to be similarly insensitive to transistor gain variations; while an LC-ladder implementation relying on beta-multiplication of an emitter impedance [11] might be more sensitive than the LC-ladder and capacitive shunt-shunt feedback technique. It should be noted, though, that the process variations of passive components can be quite high and as such all techniques should receive due consideration. Unfortunately the results of this work do not provide a clear means of improving the sensitivity of designs using these configurations as is, since the design process and final component values are determined by the LNA specifications.

The objective of this work was to find out which group of HICUM model parameters causes the highest sensitivity in the designed LNAs, and thus needs to be extracted with the highest accuracy. It also indicates the sensitivity of the LNA performance to other model parameters where the required accuracy may not be as high. This work, therefore, presents the sensitivity of LNA performance to model parameters which may have been extracted inaccurately, and also provides 
valuable information in determining what level of accuracy that should be required for each parameter.

It would also be interesting to determine which process variations have the largest impact on the performance of these LNAs. The investigation of these effects requires the accurate representation of model parameter correlation to common process parameters. The associated results will indicate which process variations are most crucial to reduce and also indicate where the configurations should be extended to make them more robust to such variations. This can be investigated in a future study. This work should also be extended to include the sensitivity of other common circuits like voltage controlled oscillators and power amplifiers to determine which parameters are the most sensitive in general.

\section{References}

[1] Schröter, M., and Chakravorty, A. (2010), Compact hierarchical modeling of bipolar transistors with HICUM, World Scientific, Singapore, ISBN 978-981-4273-21-3, 2010 (http://www.worldscibooks.com/catalogues/asset.pdf).

[2] Zimmermann, Y., Moebus, K. E., Wittkopf, H., and Schröter, M. (2009), 'TRADICA - an integrated modeling tool linking process and circuit design', Proc. IEEE Semiconductor Conference, Dresden.

[3] Voinigescu, S. P., Maliepaard, M. C., Showell, J. L., Babcock, G. E., Marchesan, D., Schröter, M., Schvan, P., and Harame, D. L. (1997), 'A Scalable Highfrequency Noise Model for Bipolar Transistors with Application to Optimal Transistor Sizing for Low-Noise Amplifier Design', IEEE Journal of Solid-State Circuits, vol. 32, no. 9, pp. 1430-1439.

[4] Lin, Y., Chen, H., Wang, T., Lin, Y., and Lu, S. (2007), '3-10-GHz Ultra-Wideband LowNoise Amplifier Utilizing Miller Effect and Inductive Shunt-Shunt Feedback Technique,' IEEE Transactions on Microwave Theory and Techniques, vol. 55, no. 9, pp. 1832-1843.

[5] Ismail, A., and Abidi, A. A. (2004), 'A 3-10-GHz low-noise amplifier with wideband LCladder matching network', IEEE Journal of Solid-State Circuits, vol. 39, no. 12, pp. 22692277.

[6] Weststrate, M., and Sinha, S. (2009), 'Mathematical Modelling of the LC-Ladder and Capacitive Shunt-Shunt Feedback LNA Topology', SAIEE Africa Research Journal, vol. 100, pp. 72-78.

[7] Weststrate, M., and Sinha, S. (2009), 'Mathematical Analysis of Input Matching Techniques With Application in Wide-band LNA Design', Proc. of the South African Conference on Semi- and Superconductor Technology, Stellenbosch, pp. 128-132.

[8] Weststrate, M., and Sinha, S. (2009), 'Noise optimization of a wideband capacitive shuntshunt feedback LNA design suitable for software-defined radio', Proc. of the IEEE International Conference on Electronics, Circuits and Systems (ICECS), Hammamet. 
[9] Min, B., and Rebeiz, G. M. (2007), 'Ka-band SiGe HBT Low Noise Amplifier Design for Simultaneous Noise and Input Power Matching,' IEEE Microwave and Wireless Components Letters, vol. 17, no. 12, pp. 891-893.

[10] Gray, P. R., Hurst, P. J., Lewis, S. H., and Meyer, R. G., (2001), Analysis and design of analog integrated circuits, 4 th ed.: John Wiley \& Sons Inc.

[11] Ismail, A. and Abidi, A. A. (2004), 'A 3-10-GHz low-noise amplifier with wideband LC-ladder matching network,' IEEE Journal of Solid-State Circuits, vol. 39, no. 12, pp. 2269-2277. 